\title{
Procollagen-Proline Dioxygenase
}

National Cancer Institute

\section{Source}

National Cancer Institute. Procollagen-Proline Dioxygenase. NCI Thesaurus. Code C116402.

A tetrameric enzyme complex that catalyzes the post-translational formation of 4hydroxyproline in the amino acid sequence -Xaa-Pro-Gly-, which is found in collagens and other proteins. The enzyme complex is comprised of two alpha chains and two beta chains. There are 3 separate genes that encode 3 distinct alpha proteins, prolyl 4hydroxylase subunit alpha-1, -2 and -3 . The beta chain is protein disulfide-isomerase. 